\title{
Temporal analysis of localization and trafficking of glycolipids
}

Kenta Arai ${ }^{1}$, Kazuya Kabayama ${ }^{1,4^{*}}$, Yoshimi Kanie ${ }^{2}$, Osamu Kanie ${ }^{3}$, and Koichi Fukase ${ }^{1,4^{*}}$

${ }^{1}$ Dept. of Chem., Grad. Sch. of Sci., Osaka University, Toyonaka, JAPAN.

${ }^{2}$ Research Promotion Division, Tokai university, Hiratsuka, JAPAN.

${ }^{3}$ Sch. of Eng., Tokai University, Hiratsuka, JAPAN.

${ }^{4}$ MS-CORE, PRC, Grad. Sch. of Sci., Osaka University, Toyonaka, JAPAN.

* To whom correspondence should be addressed:

Kazuya Kabayama, Dept. of Chem., Grad. Sch. of Sci., Osaka University, Toyonaka, JAPAN.

Tel: (+81)-6-6850-5192, Fax: (+81)-6-6850-5419, e-mail: kaba@chem.sci.osaka-u.ac.jp

Koichi Fukase, Dept. of Chem., Grad. Sch. of Sci., Osaka University, Toyonaka, JAPAN.

Tel: (+81)-6-6850-5388, Fax: (+81)-6-6850-5419, e-mail: koichi@chem.sci.osaka-u.ac.jp 
Journal of Lipid Research Arai et al.

Key words:

Glycolipids, Transport, Sphingolipids, Fluorescence microscopy, Golgi apparatus, Live cell imaging

\section{Abbreviation list}

LacCer; lactosyl ceramide, TGN; trans-Golgi network, BSA; bovine serum albumin, THF;

tetrahydrofuran, DMT-MM; 4-(4,6-Dimethoxy-1,3,5-triazin-2-yl)-4-methylmorpholinium

chloride, D-PBS; Dulbecco's phosphate-buffered saline, FBS; fetal bovine serum, GalNAcT2; N-

acetylgalactosaminyl-transferase 2, STX6; syntaxin 6, MS; mass spectrometry, LC-MS; liquid

chromatography-mass spectrometry, PMT; photomultiplier tube, GaAsP; Gallium arsenide

phosphide, ER; endoplasmic reticulum, GlcCer; glucosyl ceramide, Cer; ceramide 
Journal of Lipid Research Arai et al.

\section{Abstract}

Glycolipid metabolism occurs in the Golgi apparatus, but the detailed mechanisms have not yet

been elucidated. We used fluorescently labeled glycolipids to analyze glycolipid composition and

localization changes and shed light on glycolipid metabolism. In a previous study, the fatty chain

of lactosyl ceramide was fluorescently labeled with BODIPY (LacCer-BODIPY) before being

introduced into cultured cells to analyze the cell membrane glycolipid recycling process. However,

imaging analysis of glycolipid recycling is complicated because of limited spatial resolution.

Therefore, we examined the microscopic conditions that allow the temporal analysis of LacCer-

BODIPY trafficking and localization. We observed that the glycolipid fluorescent probe migrated

from the cell membrane to intracellular organelles before returning to the cell membrane. We

used confocal microscopy to observe co-localization of the glycolipid probe with endosomes and

Golgi markers, demonstrating that it recycles mainly through the trans-Golgi network (TGN).

Here, a glycolipid recycling pathway was observed that did not require the lipids to pass through

the lysosome. 
Journal of Lipid Research Arai et al.

\section{Introduction}

The homeostasis of glycolipids expressed on the cell membrane is maintained by a balance

between de novo synthesis and degradation and recycling (1-3). Several studies have

biochemically analyzed these pathways using inhibitors $(4,5)$. Pagano et al. performed a spatio-

temporal analysis of glycolipid metabolism by adding fluorescently labeled glycolipids to cells

and fractionating organelles. They used fluorescently labeled lactosyl ceramide (LacCer-

BODIPY, Fig. 1A) in which the fatty acid chain was replaced with a 5-carbon alkyl chain linked

to the fluorescent probe BODIPY. They confirmed that this probe was taken up by cells via the

same route as radiolabeled LacCer (6-12). These reports demonstrated that glycolipids

internalized from the cell membrane localize to the Golgi apparatus and lysosomes before some

glycolipids are recycled back to the cell membrane. However, a comprehensive spatial and

temporal understanding of the transported glycolipids has not yet been achieved due to differences

in the conditions and analytical methods of adding these glycolipid derivatives.

We previously constructed a system to spatio-temporally analyze glycolipid metabolism using

LacCer-BODIPY (13-15). We used this exogenous glycolipid probe to perform a detailed time-

lapse analysis of the recycling pathway. Here, we successfully visualized a transport pathway that 
translocates glycolipids to the Golgi without them having to pass through the lysosome and whereby they quickly return to the cell membrane.

\section{Experimental section}

\section{LacCer-BODIPY synthesis and BSA complex preparation}

Details of the synthesis process are described in our previous report (16). Briefly, $3.0 \mu \mathrm{mol} \beta$ -

D-galactopyranosyl -(1->4)- $\beta$-D-glucopyranosyl-(1->1')-D-erythro-sphingosine was dissolved in $200 \mu \mathrm{L}$ tetrahydrofuran (THF)/ $\mathrm{H}_{2} \mathrm{O}(3: 1 \mathrm{v} / \mathrm{v})$ and mixed with $3.0 \mu \mathrm{mol}$ BODIPY $\mathrm{C}_{5}$ (Thermo

Fisher Scientific, Waltham, MA, USA) dissolved in $100 \mu \mathrm{L}$ of THF/ $\mathrm{H}_{2} \mathrm{O}(3: 1 \mathrm{v} / \mathrm{v}) .12 .3 \mu \mathrm{mol} 4$ -

(4,6-Dimethoxy-1,3,5-triazin-2-yl)-4-methylmorpholinium chloride (DMT-MM; Tokyo Kasei

Inc. Tokyo, Japan) was added, and the mixture was stirred for $2 \mathrm{~h}$ at $40^{\circ} \mathrm{C}$. The product was subsequently purified using preparative thin-layer chromatography. Dried LacCer-BODIPY was dissolved in $200 \mu \mathrm{L}$ of ethanol and added dropwise to $4 \mathrm{~mL}$ of Dulbecco's phosphate-buffered saline (D-PBS) containing fatty acid-free bovine serum albumin (BSA; Sigma-Aldrich Corp. St. Louis, MO, USA) while vortexing. The complex was dialyzed overnight at $4^{\circ} \mathrm{C}$ against $500 \mathrm{~mL}$ 
Journal of Lipid Research Arai et al.

of D-PBS and ultra-centrifuged $(100,000 \times \mathrm{g})$ at $4^{\circ} \mathrm{C}$ for $20 \mathrm{~min}$. Next, the supernatant (LacCer-

BODIPY-BSA complex) was collected and stored at $-20^{\circ} \mathrm{C}$ in $1.5 \mathrm{~mL}$ tubes.

\section{Cell culture}

CHO-K1 cells were cultured in Ham's F-12 (Wako, Tokyo, Japan) containing 10\% fetal bovine serum (FBS; Thermo Fisher Scientific) and 1\% penicillin-streptomycin solution $\times 100$ (Wako) in culture dishes (Greiner, Kremsmünster, Austria). Cells were passaged at a density of $80 \%$ using $0.25 \mathrm{w} / \mathrm{v} \%$ Trypsin-1 mM EDTA $4 \mathrm{Na}$ solution with phenol red (Wako) at two- to three-day intervals.

\section{Pulse chase method}

CHO-K1 cells were cultured in $35 \mathrm{~mm}$ glass-bottom dishes (IWAKI, Tokyo, Japan) to $60 \%$ confluency. The LacCer-BODIPY-BSA complex was diluted with cell culture medium to prepare a $2.5 \mu \mathrm{M}$ solution. After washing the cells once with saline, $2.5 \mu \mathrm{M}$ LacCer-BODIPY-BSA 
complex was added dropwise and incubated at $23^{\circ} \mathrm{C}$ for $1 \mathrm{~min}$. Next, $2 \mathrm{~mL}$ of medium was added

after washing twice with saline.

\section{Live cell imaging}

Lysosomes were stained with LysoTracker ${ }^{\circledR}$ Red DND-99 (Thermo Fisher Scientific) for 5 min.

Early endosomes were stained with CellLight ${ }^{\circledR}$ Early Endosomes-RFP (Rab5a-RFP) and BacMam 2.0 overnight. Golgi stacks were stained with CellLight ${ }^{\circ}$ Golgi-RFP (Nacetylgalactosaminyl-transferase 2 (GalNAcT2-RFP), BacMam 2.0, or CellLight ${ }^{\circ}$ Golgi-GFP (GalNAcT2-GFP), and BacMam 2.0 (Thermo Fisher Scientific) overnight, respectively. The plasmid of human syntaxin 6-mCherry $\quad$ (pRP[Exp]-NeoCMV>hSTX6[NM_005819.5](ns):mCherry) (VectorBuilder, Chicago, IL， USA) was constructed and introduced into cells using Lipofectamine ${ }^{\circledR} 2000$ (Thermo Fisher Scientific) to stain TGN. LacCer-BODIPY was pulsed into cells and chased using time-lapse imaging with a

Nikon A1R (Nikon, Tokyo, Japan) at $37^{\circ} \mathrm{C}$ in a $5 \% \mathrm{CO}_{2}$ atmosphere.

\section{Confocal microscopy}


Journal of Lipid Research Arai et al.

The confocal microscope OLYMPUS FV1000-D (OLYMPUS, Tokyo, Japan) equipped with a temperature control stage $\left(37^{\circ} \mathrm{C}\right)$ and UPLSAPO x60/N.A. 1.20 water immersion objective was used. Excitations were set to $488 \mathrm{~nm}$, and emissions were collected at $519 \mathrm{~nm}$. The confocal microscope Nikon A1R (Nikon, Tokyo, Japan) equipped with a temperature control stage $\left(37^{\circ} \mathrm{C}\right)$, Plan Apo VC 60x A WI/N.A. 1.20 water immersion objective was also used for time-lapse imaging. Excitations were set to $488 \mathrm{~nm}$ and $561 \mathrm{~nm}$, and emissions were collected at 525-575 $\mathrm{nm}$ and 595-645 $\mathrm{nm}$.

\section{Results}

\section{Fluorescently labeled LacCer-BODIPY glycolipid is recycled}

In vivo, the homeostasis of glycolipids includes de novo synthesis and multiple recycling

pathways. However, the details of these processes are not yet completely understood. Therefore, 
Journal of Lipid Research Arai et al.

we examined the uptake of labeled glycolipids in living cells, their incorporation into the cell

membrane, and their subsequent trafficking within the cells. For this purpose, we developed a

live-cell imaging method to visualize glycolipid recycling. Conventional methods of intracellular

trafficking analysis using LacCer-BODIPY (Fig. 1A) apply temperature control to accumulate

the probe in the cell and facilitate microscopic observation and mass spectrometry (MS) analysis.

However, tracking probe localization changes in the cell has been difficult. Therefore, we tracked

glycolipid probe localization by improving the time resolution for $1 \mathrm{~min}$ (pulse) and chasing the

probe. Under these conditions, the intracellular localization of the probe was observed for a period

from 5 to $120 \mathrm{~min}$ (Fig. 1B).

After adding the probe, the cells were incubated for 5, 15, 30, 60, 100, and $120 \mathrm{~min}$ and observed

under a microscope. Localization on the cell membrane was confirmed at 5 min after LacCer-

BODIPY incorporation, and vesicle-like fluorescence aggregation was directly observed under

the cell membrane after $15 \mathrm{~min}$. The fluorescent probe was observed to be localized near the

nucleus in samples incubated for 30 to $100 \mathrm{~min}$.

Furthermore, localized fluorescent molecules could not be observed on the cell membrane after

$100 \mathrm{~min}$ of incubation. However, the fluorescent probe localized to the cell membrane after 
Journal of Lipid Research Arai et al.

incubation for $120 \mathrm{~min}$. These results indicate that the glycolipid probe recycles from the cell

membrane to intracellular organelles and back to the cell surface once every two hours.

\section{LacCer-BODIPY transport depends on its concentration}

We previously showed that LacCer-BODIPY is intracellularly metabolized and undergoes

glycosylation and degradation (15). In recent years, we have analyzed structural changes in the

probe using liquid chromatography-mass spectrometry (LC-MS) by adding LacCer-BODIPY to

CHO-K1 cells for $1 \mathrm{~min}$ (pulse) and chasing for $10 \mathrm{~min}$ (16). This report showed that the probe

addition conditions caused differences in glycosylation.

Therefore, we examined the localization of the probe using lysosomal markers following a 30-

min chase. Only $5.6 \%$ of the probes localized to lysosomes following a 1-min pulse with LacCer-

BODIPY and a chase for $30 \mathrm{~min}$. On the other hand, $28.1 \%$ of the probes localized to lysosomes

following a 10-min pulse and a 30-min chase (Fig. 2).

This experiment revealed that probe localization is dose-dependent, and this was previously

shown using LC-MS analysis. Therefore, these results combined with those presented in Figure 
Journal of Lipid Research Arai et al.

1B suggest that a 1-min pulse with this probe could help visualize the recycling pathway without

the lipids being targeted to the degradation pathway.

\section{Development of a time-lapse imaging method to study LacCer-BODIPY recycling}

Time-lapse imaging was performed to examine LacCer-BODIPY recycling in more detail.

Conventional observation techniques do not allow continuous observation of the trafficking

process because of the fading of BODIPY fluorescence. We used a high-sensitivity

photomultiplier tube (PMT), which uses gallium arsenide phosphide (GaAsP) as the

photosensitive compound, to suppress fluorescence fading. A photocathode device was the

detector in the confocal laser scanning microscope, and the excitation laser power was set as low

as possible. We continuously observed glycolipid recycling in one cell by fixing the observation

area. Immediately after pulse labeling the cells with LacCer-BODIPY, imaging began at $15 \mathrm{~min}$

intervals.

Similar to the results in Figure 1B, it was confirmed that the probe aggregated in the nuclear

periphery after $30 \mathrm{~min}$. Thereafter, the probe could not be observed on the cell membrane until 
Journal of Lipid Research Arai et al.

$90 \mathrm{~min}$, but it was identified on the cell membrane at $120 \mathrm{~min}$ (Fig. 3). The original movie can

be found in the supplemental data (Supplemental movie S1).

\section{LacCer-BODIPY is transported to the TGN via early endosomes}

Next, the localization to the early endosome, which is important for endocytosis, was analyzed

using time-lapse imaging. It was confirmed that Rab5a-RFP, which is known to localize to early

endosomes, and LacCer-BODIPY co-localized after a 30-min chase (Fig. 4A). Furthermore, the

same method expressed GalNAcT2-RFP, which is known to be localized in Golgi stacks, in cells.

Moreover, its localization with LacCer-BODIPY was examined, but their co-localization was not

observed after $60 \mathrm{~min}$ and $90 \mathrm{~min}$ (Fig. 4B).

Therefore, we expressed human syntaxin 6 , which is known to localize to TGN, fused to

mCherry (STX6-mCherry), and examined its co-localization with LacCer-BODIPY. After 60-

min and 90-min chases, co-localization of STX6-mCherry and LacCer-BODIPY was observed

(Fig. 4C). These results indicated that LacCer-BODIPY was recycled from the early endosome

to the plasma membrane via TGN. Furthermore, we also observed that GalNAcT2-GFP and

STX6-mCherry did not co-localize (Fig. 4D). 
Journal of Lipid Research Arai et al.

\section{Discussion}

We analyzed the glycolipid transport pathway in more detail using LacCer-BODIPY, which is

known as an analysis probe of the glycolipid transport pathway. We successfully visualized the

details of glycolipid transport after being taken up from the cell membrane, and this revealed their

recycling (Fig. 1B, 3).

Various transport analyses using this probe have been studied by other researchers. In the study

of lipid accumulation disease using Drosophila, glycolipid uptake by neurons and analysis of

their distribution in cells using LacCer-BODIPY have been reported. This study suggested that

cholesterol depletion inhibits the transport of glycolipids to the Golgi and ER and promotes their

degradation. However, the exact transport pathway in mammals has not been clarified since

LacCer is not present in Drosophila (17).

Other studies have used mammalian cells. Hoekstra et al. observed that fluorescently labeled

glycolipids incorporated into BHK-21 cells using liposomes were endocytosed and returned to

the cell membrane (18). Pagano et al. used BSA to remove residual probes from cell membranes

and elucidated the probe transport pathway from early endosomes. However, it was not possible 
Journal of Lipid Research Arai et al.

to subdivide the recycling route (12). Here, we first improved the temporal resolution by controlling the amount of probe taken up using a pulse chase (Fig. 1B). It was observed that probe recycling between the cell membrane and the organelle occurred in $2 \mathrm{~h}$ (Fig. 1B, 3). In addition, we found that the transport pathway occurs via TGN only in the Golgi apparatus (Fig. 4B-D).

A pathway for the intracellular transport and metabolism of externally taken up glycolipids, involving degradation via lysosomes, has been confirmed in studies using radioisotope-labeled glycolipids (19). Observation of metabolism by glycolipid uptake for 0.5 to $4 \mathrm{~h}$ confirmed that most of the introduced glycolipids were transported to lysosomes for degradation (20, 21). Here, we examined glycolipid uptake in a shorter time $(1 \mathrm{~min})$. There was less transport of probes to lysosomes with this method. This is the result of a very small amount of tracking compared to previous uptake methods (Fig. 2A left). This is inferred from the fact that, in the previously reported LC-MS study, lysosome-mediated sphingomyelin synthesized by lysosomes was detected by a 10-min pulse, but it was hardly detected by a 1-min pulse (16).

BODIPY is a fluorescent probe that is relatively easy to photobleach. Therefore, it was necessary to reduce the excitation laser power as much as possible and improve the sensitivity of the detector to obtain long-term time-lapse imaging (Fig. 3). We often use a multi-alkali photocathode for the 
Journal of Lipid Research Arai et al.

PMT of confocal microscopes. Here, we used the high-sensitivity PMT, GaAsP, which is about ten times more sensitive than a standard PMT (22). This improvement could help detect a relatively small amount of the probe for a long time without significant photobleaching, and this clarified the recycling pathway. Using a similar concept, we previously imaged fluorescently labeled ligands of Toll-like receptor 2 diluted to physiologically active concentrations and successfully visualized receptor expression-dependent endocytosis (23).

The intracellular transport pathway of LacCer-BODIPY identified here is similar to the glycoprotein and glycolipid transport pathways previously investigated using the B subunit of cholera or Shiga toxin $(24,25)$. These toxins require STX6 for transport and are known to localize to early endosomes before TGN. We demonstrated that LacCer-BODIPY is also transported by a similar pathway. In addition, we were the first to successfully visualize that transport from early endosomes is transferred to the TGN without passing through Golgi stacks.

In conclusion, we visualized the recycling pathway of exogenous glycolipids from early endosomes to TGN by introducing LacCer-BODIPY into cells with short pulses (Fig. 5A). In the previously reported studies, since the glycolipids were taken up in a longer time than the 1-min pulse shown here, we considered that the excess fluorescently labeled glycolipids were 
Journal of Lipid Research Arai et al.

transported to lysosomes (Fig. 5B). We visualized the return of lipids to the plasma membrane

using time-lapse imaging with this probe. The pathway shown in Figure 5A is likely to rapidly

and reasonably return lipids to the plasma membrane to maintain cell membrane homeostasis.

\section{Acknowledgements}

This work was supported by Grants-in-Aid for Scientific Research, KAKENHI (K. K.; JP

23770155, JP18K05356; K. F.; JP16H01885; O. K.; JP15K14399), by the MEXT-Supported

Program (JSPS) for the Strategic Research Foundation at Private Universities (K. K.; S1411010).

We would like to thank Editage (www.editage.com) for English language editing. 
Journal of Lipid Research Arai et al.

\section{References}

1. Groener, J. E., W. Bax, and B. J. Poorthuis. 1996. Metabolic fate of oleic acid

derived from lysosomal degradation of cholesteryl oleate in human fibroblasts. J Lipid Res $\mathbf{3 7}$ :

2271-2279.

2. Chatelut, M., M. Leruth, K. Harzer, A. Dagan, S. Marchesini, S. Gatt, R. Salvayre, P.

Courtoy, and T. Levade. 1998. Natural ceramide is unable to escape the lysosome, in contrast to

a fluorescent analogue. FEBS Lett 426: 102-106.

3. Sillence, D. J., and F. M. Platt. 2004. Glycosphingolipids in endocytic membrane

transport. Semin Cell Dev Biol 15: 409-416.

4. Gillard, B. K., R. G. Harrell, and D. M. Marcus. 1996. Pathways of

glycosphingolipid biosynthesis in SW13 cells in the presence and absence of vimentin

intermediate filaments. Glycobiology 6: 33-42.

5. Gillard, B. K., R. G. Clement, and D. M. Marcus. 1998. Variations among cell lines

in the synthesis of sphingolipids in de novo and recycling pathways. Glycobiology 8: 885-890.

6. Chen, C. S., G. Bach, and R. E. Pagano. 1998. Abnormal transport along the

lysosomal pathway in mucolipidosis, type IV disease. Proc Natl Acad Sci U S A 95: 6373-6378. 
Journal of Lipid Research Arai et al.

7. Puri, V., R. Watanabe, R. D. Singh, M. Dominguez, J. C. Brown, C. L. Wheatley, D.

L. Marks, and R. E. Pagano. 2001. Clathrin-dependent and -independent internalization of

plasma membrane sphingolipids initiates two Golgi targeting pathways. J Cell Biol 154: 535-

547.

8. $\quad$ Benmerah, A., M. Bayrou, N. Cerf-Bensussan, and A. Dautry-Varsat. 1999.

Inhibition of clathrin-coated pit assembly by an Eps15 mutant. J Cell Sci 112 ( Pt 9): 1303-

1311.

9. Singh, R. D., Y. Liu, C. L. Wheatley, E. L. Holicky, A. Makino, D. L. Marks, T.

Kobayashi, G. Subramaniam, R. Bittman, and R. E. Pagano. 2006. Caveolar endocytosis and

microdomain association of a glycosphingolipid analog is dependent on its sphingosine

stereochemistry. J Biol Chem 281: 30660-30668.

10. Singh, R. D., V. Puri, J. T. Valiyaveettil, D. L. Marks, R. Bittman, and R. E. Pagano.

2003. Selective caveolin-1-dependent endocytosis of glycosphingolipids. Mol Biol Cell 14:

$3254-3265$.

11. Pagano, R. E., and R. G. Sleight. 1985. Defining lipid transport pathways in animal

cells. Science 229: 1051-1057. 
Journal of Lipid Research Arai et al.

12. Marks, D. L., R. Bittman, and R. E. Pagano. 2008. Use of Bodipy-labeled

sphingolipid and cholesterol analogs to examine membrane microdomains in cells. Histochem

Cell Biol 130: 819-832.

13. Son, S. H., S. Daikoku, A. Ohtake, K. Suzuki, K. Kabayama, Y. Ito, and O. Kanie.

2014. Syntheses of lactosyl ceramide analogues carrying novel bifunctional BODIPY dyes

directed towards the differential analysis of multiplexed glycosphingolipids by MS/MS using

iTRAQ. Chem Commun (Camb) 50: 3010-3013.

14. Daikoku, S., Y. Ono, A. Ohtake, Y. Hasegawa, E. Fukusaki, K. Suzuki, Y. Ito, S.

Goto, and O. Kanie. 2011. Fluorescence-monitored zero dead-volume nanoLC-microESI-QIT-

TOF MS for analysis of fluorescently tagged glycosphingolipids. Analyst 136: 1046-1050.

15. Ohtake, A., S. Daikoku, K. Suzuki, Y. Ito, and O. Kanie. 2013. Analysis of the

cellular dynamics of fluorescently tagged glycosphingolipids by using a nanoliquid

chromatography-tandem mass spectrometry platform. Anal Chem 85: 8475-8482.

16. Kanie, Y., M. Taniuchi, and O. Kanie. 2018. Evaluation of reversed-phase nano

liquid chromatography conditions by using reversed-phase thin layer chromatography based on 
Journal of Lipid Research Arai et al.

Hansen solubility parameters for the analysis of amphiphilic glycosylsphingolipid

transformations. J Chromatogr A 1534: 123-129.

17. Hortsch, R., E. Lee, N. Erathodiyil, S. Hebbar, S. Steinert, J. Y. Lee, D. S. Chua, and

R. Kraut. 2010. Glycolipid trafficking in Drosophila undergoes pathway switching in response

to aberrant cholesterol levels. Mol Biol Cell 21: 778-790.

18. Kok, J. W., S. Eskelinen, K. Hoekstra, and D. Hoekstra. 1989. Salvage of

glucosylceramide by recycling after internalization along the pathway of receptor-mediated

endocytosis. Proc Natl Acad Sci U S A 86: 9896-9900.

19. Tettamanti, G., R. Bassi, P. Viani, and L. Riboni. 2003. Salvage pathways in

glycosphingolipid metabolism. Biochimie 85: 423-437.

20. Riboni, L., R. Bassi, A. Prinetti, and G. Tettamanti. 1996. Salvage of catabolic

products in ganglioside metabolism: a study on rat cerebellar granule cells in culture. FEBS Lett

391: $336-340$.

21. Ghidoni, R., L. Riboni, and G. Tettamanti. 1989. Metabolism of exogenous

gangliosides in cerebellar granule cells, differentiated in culture. J Neurochem 53: 1567-1574. 
Journal of Lipid Research Arai et al.

22. Niigaki, M., T. Hirohata, T. Suzuki, H. Kan, and T. Hiruma. 1997. Field-assisted

photoemission from InP/InGaAsP photocathode with p/n junction. Appl Phys Lett 71: 2493-

2495.

23. Arai, Y., K. Yokoyama, Y. Kawahara, Q. Feng, I. Ohta, A. Shimoyama, S. Inuki, K.

Fukase, K. Kabayama, and Y. Fujimoto. 2018. Time-lapse monitoring of TLR2 ligand

internalization with newly developed fluorescent probes. Org Biomol Chem 16: 3824-3830.

24. Pfeffer, S. R. 2011. Entry at the trans-Face of the Golgi. Csh Perspect Biol 3.

25. Ganley, I. G., E. Espinosa, and S. R. Pfeffer. 2008. A syntaxin 10-SNARE complex

distinguishes two distinct transport routes from endosomes to the trans-Golgi in human cells. $J$

Cell Biol 180: 159-172. 
Journal of Lipid Research Arai et al.

\section{Figure legends}

Figure 1. Time-dependent localization of LacCer-BODIPY using pulse chase in CHO-K1 cells.

A) Structure of BODIPY $\mathrm{C}_{5}$ labeled lactosyl ceramide (LacCer-BODIPY). B) Confocal

microscope images of LacCer-BODIPY localization when incubated at 5, 15, 30, 60, 100, and

120 min after 1-min pulse labeling. The lower panels show enlargement of the area within the

white-box of the upper panels. Scale bar; $10 \mu \mathrm{m}$.

Figure 2. Localization analysis of LacCer-BODIPY with lysosomal markers.

A) Localized observation of LacCer-BODIPY to lysosomes following pulse labeling for $1 \mathrm{~min}$

(left) and $10 \mathrm{~min}$ (right) and chase for $30 \mathrm{~min}$. The lower panels show enlargement of the area

within the white-box of the upper panels. Magenta; Lysosome, Green; LacCer-BODIPY Scale

bar; $10 \mu \mathrm{m}$. B) Co-localization ratio of LacCer-BODIPY to lysosomes. Three cells were randomly

selected from the images in this figure, and the ratios of LacCer-BODIPY localized to lysosomes

were calculated. Statistical significance was determined using a two-tailed paired t-test.

$(* p<0.003)$ 
Journal of Lipid Research Arai et al.

Figure 3. Time-lapse imaging of LacCer-BODIPY using 1-min pulse labeling.

$2.5 \mu \mathrm{M}$ of the probe was taken up into the $\mathrm{CHO}-\mathrm{K} 1$ cells by pulse labeling for $1 \mathrm{~min}$. The images

were taken with a confocal microscope equipped with a GaAsP detector 5 min after pulse labeling.

The images were taken every $15 \mathrm{~min}$ until $135 \mathrm{~min}$ after probe introduction. The images are listed

every $30 \mathrm{~min}$. The movie is posted as supplemental data. Scale bar; $10 \mu \mathrm{m}$

Figure 4. Co-localization of organelle markers and LacCer-BODIPY.

A) Localized observation of LacCer-BODIPY in the early endosome. CHO-K1 cells expressing

the Rab5a-RFP were pulse-labeled with LacCer-BODIPY and observed after 30 min using

confocal microscopy. Magenta; Rab5a-RFP, green; LacCer-BODIPY. B, C) Localized

observation of LacCer-BODIPY to the Golgi apparatus. GalNAcT2-RFP expressing cells (B) or

STX6-mCherry expressing cells (C) were pulse-labeled with LacCer-BODIPY, and time-lapse

observations were performed using confocal microscopy. Magenta; GalNAcT2-RFP (B), STX6-

mCherry (C), Green; LacCer-BODIPY. The white arrow indicates co-localization where the pixel 
Journal of Lipid Research Arai et al.

color is white. D) Staining of Golgi apparatus with STX6-mCherry (magenta) and GalNAcT2-

GFP (green). The view was obtained by performing a standard deviation projection of a Z-stack

in ImageJ. Scale bar; $10 \mu \mathrm{m}$.

Figure 5. Diagram of the proposed transport pathway for LacCer on plasma membrane.

A) Up to a certain amount of LacCer on plasma membrane is transported to early endosomes

before returning to the plasma membrane via TGN. B) When large amounts of LacCer are present

on plasma membrane, the excess LacCer is transported to lysosome before being degraded or

recycled. GlcCer; glucosyl ceramide, Cer; ceramide.

\section{Supplemental data}

Supplemental movie S1. Time-lapse imaging of LacCer-BODIPY intracellular tracking. 
bioRxiv preprint doi: https://doi.org/10.1101/2020.04.07.030411; this version posted April 8, 2020. The copyright holder for this preprint (which was not certified by peer review) is the author/funder, who has granted bioRxiv a license to display the preprint in perpetuity. It is made available under aCC-BY-NC-ND 4.0 International license.

Journal of Lipid Research Arai et al.

The movie of the intracellular transport of LacCer-BODIPY in CHO-K1 cells was taken using a

confocal microscope with GaAsP PMT. This movie was taken every 15 min for 135 min after

probe introduction and with automatic brightness correction. 


\section{Figure 1}

A)

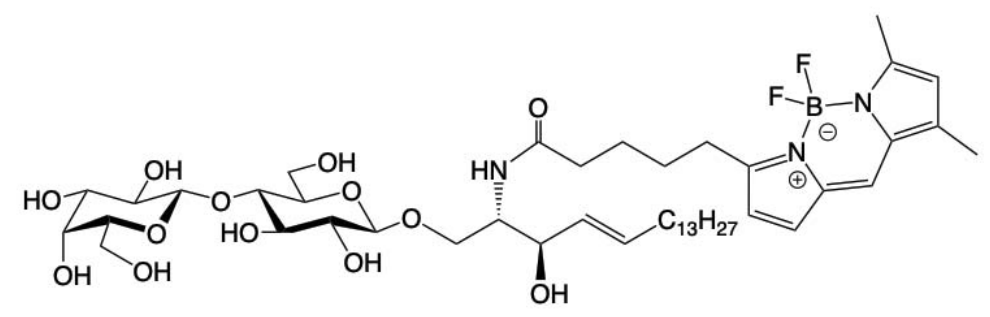

B)

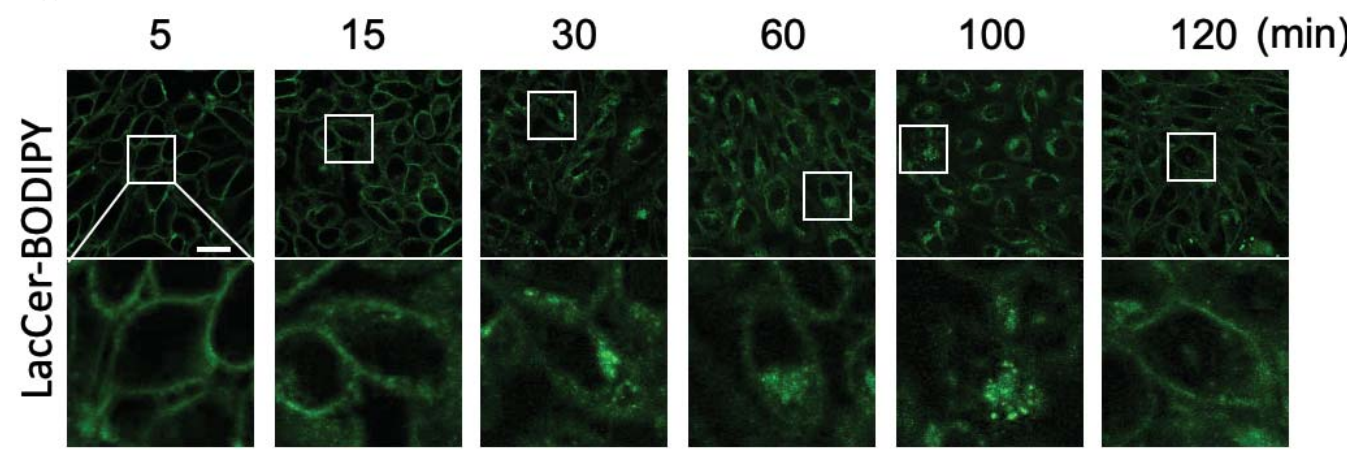


Figure 2

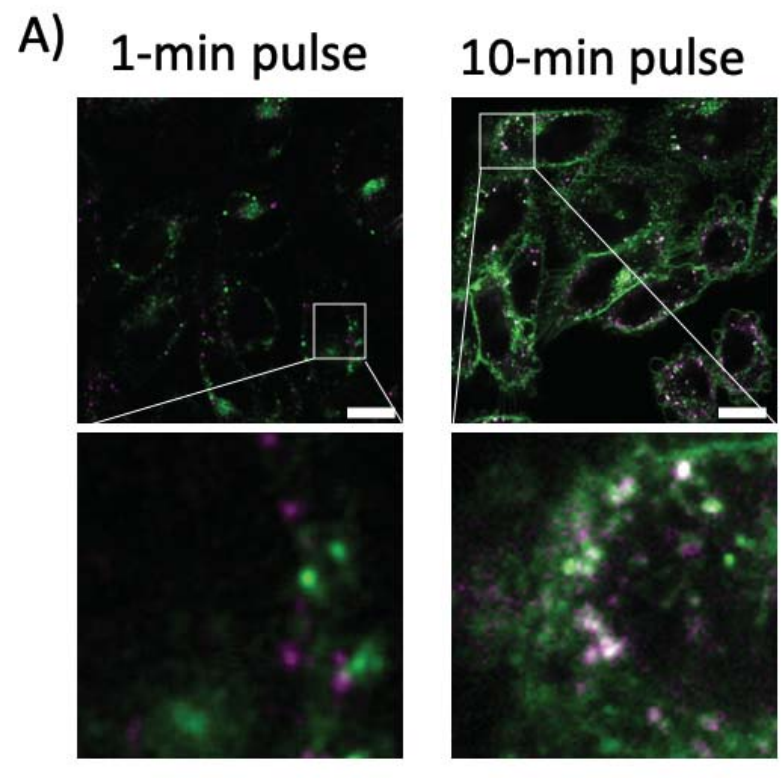

LacCer-BODIPY / Lysosome

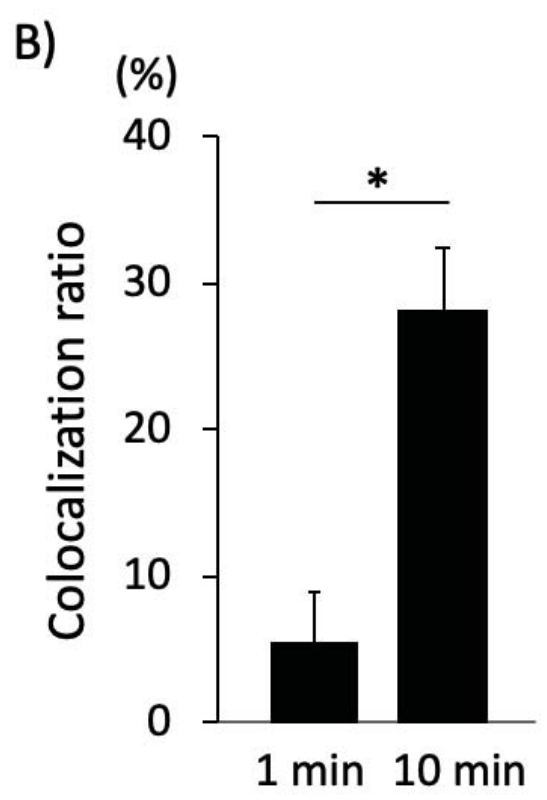


bioRxiv preprint doi: https://doi.org/10.1101/2020.04.07.030411; this version posted April 8, 2020. The copyright holder for this preprint (which was not certified by peer review) is the author/funder, who has granted bioRxiv a license to display the preprint in perpetuity. It is made available under aCC-BY-NC-ND 4.0 International license.

Journal of Lipid Research Arai et al.

Figure 3
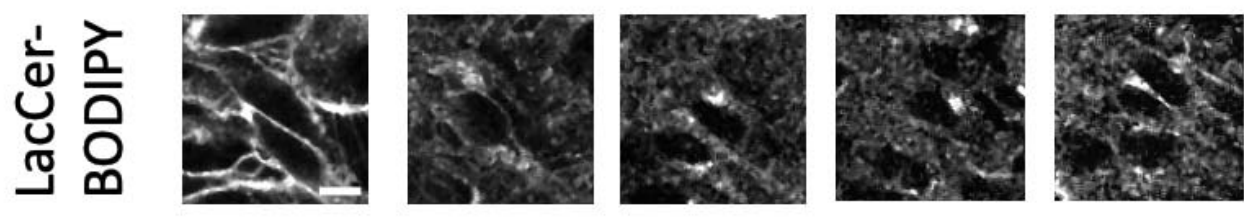

0

30

60

90

$120(\min )$ 
Figure 4

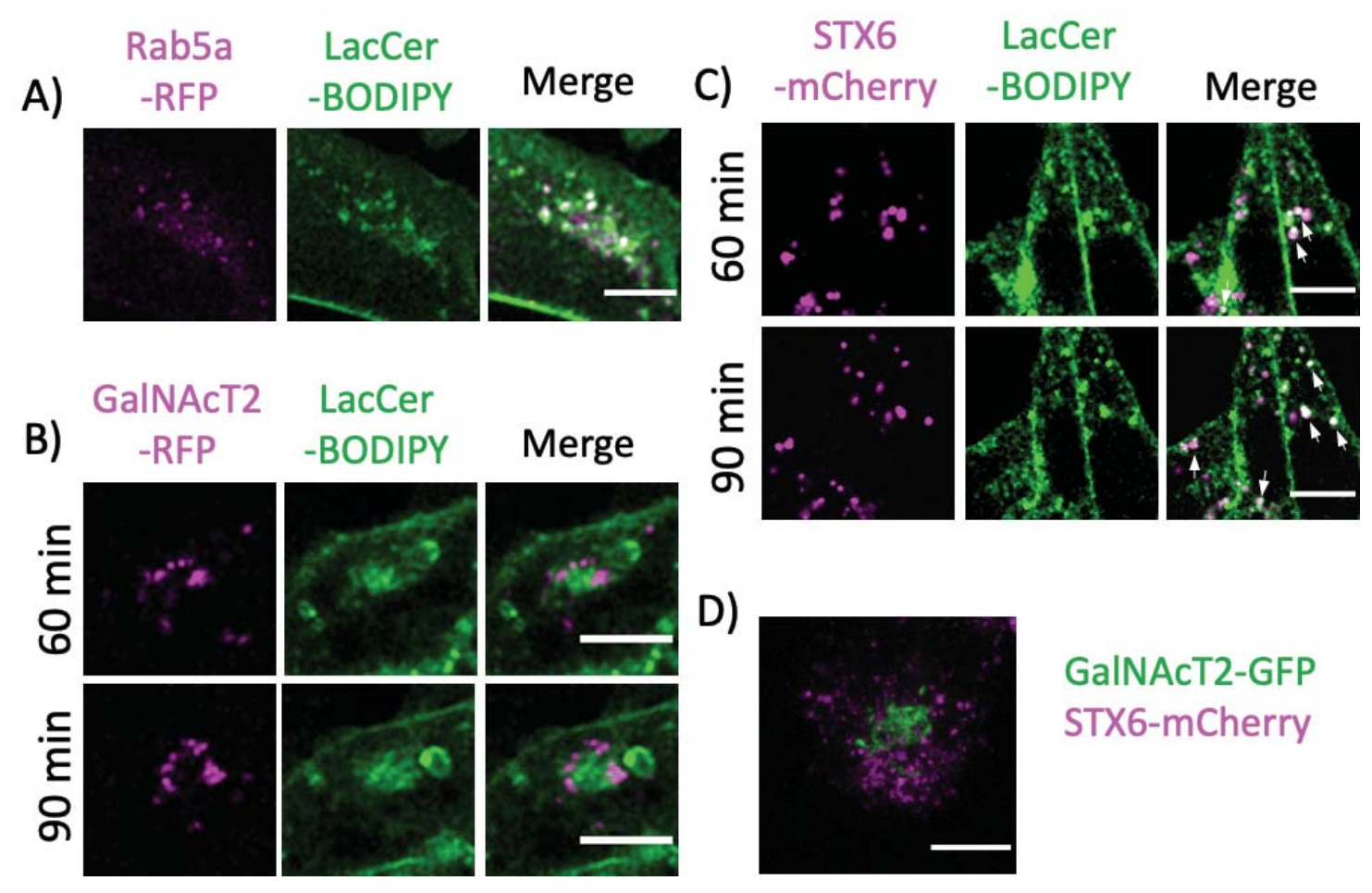


bioRxiv preprint doi: https://doi.org/10.1101/2020.04.07.030411; this version posted April 8, 2020. The copyright holder for this preprint (which was not certified by peer review) is the author/funder, who has granted bioRxiv a license to display the preprint in perpetuity. It is made available under aCC-BY-NC-ND 4.0 International license.

Journal of Lipid Research Arai et al.

Figure 5

A)

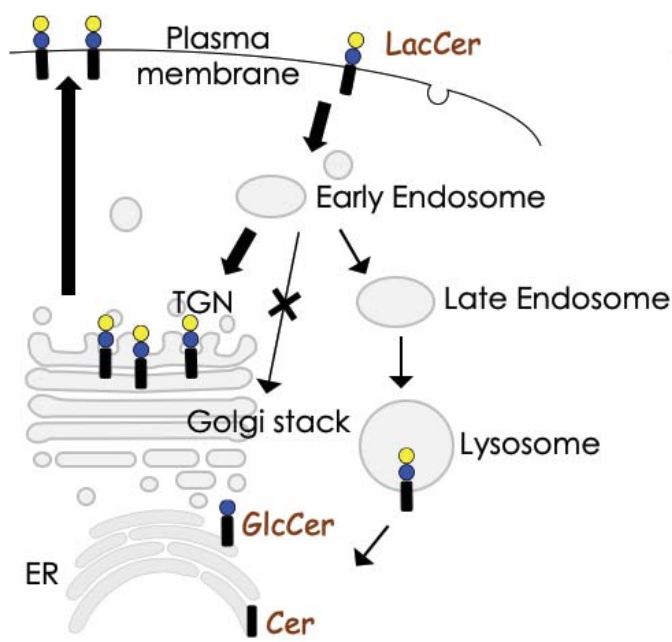

B)

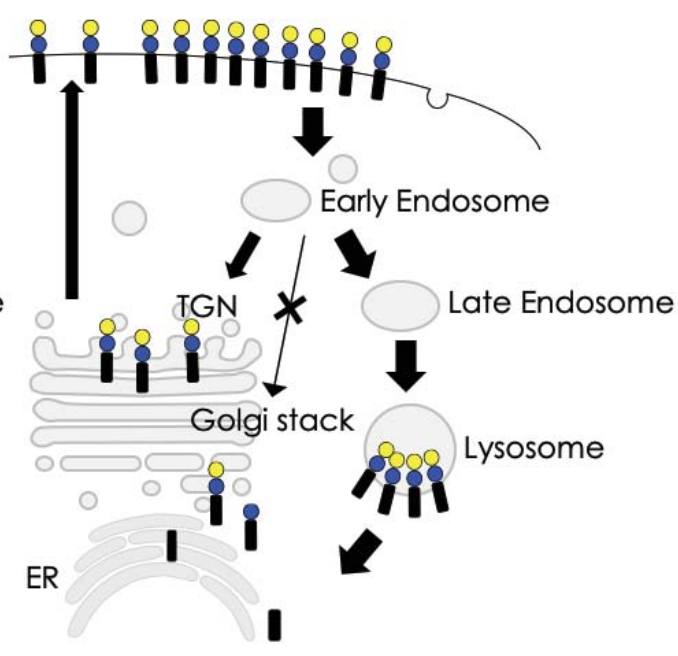

